\title{
Low Porosity Metallic Periodic Structures with Negative Poisson's Ratio
}

\section{Citation}

Taylor, Michael, Luca Francesconi, Miklós Gerendás, Ali Shanian, Carl Carson, and Katia Bertoldi. 2014. "Low Porosity Metallic Periodic Structures with Negative Poisson's Ratio." Advanced Materials 26 (15) (April): 2365-2370. doi:10.1002/adma.201304464. http:// dx.doi.org/10.1002/adma.201304464.

\section{Published Version}

doi:10.1002/adma.201304464

\section{Permanent link}

http://nrs.harvard.edu/urn-3:HUL.InstRepos:12111442

\section{Terms of Use}

This article was downloaded from Harvard University's DASH repository, and is made available under the terms and conditions applicable to Open Access Policy Articles, as set forth at http:// nrs.harvard.edu/urn-3:HUL.InstRepos:dash.current.terms-of-use\#OAP

\section{Share Your Story}

The Harvard community has made this article openly available.

Please share how this access benefits you. Submit a story.

\section{Accessibility}




\title{
Low Porosity Metallic Periodic Structures with Negative Poisson's Ratio
}

\author{
Michael Taylor *, Luca Francesconi ${ }^{\dagger}$, Miklós Gerendás ${ }^{\ddagger}$, Ali Shanian ${ }^{\S}$, Carl Carson ${ }^{\S}$, Katia Bertoldi * $\uparrow$ \\ *School of Engineering and Applied Science, Harvard University, Cambridge, MA 02138, † Department of Material Science and Mechanical Engineering, Universitá \\ degli Studi di Cagliari, Italy, ${ }^{\top}$ Kavli Institute, Harvard University, Cambridge, MA 02138, ${ }^{\ddagger}$ Rolls-Royce Deutschland Ltd \& Co KG, Dahlewitz, 15827 Blankenfelde-Mahlow, \\ Germany, and § Rolls-Royce Energy, 9545 Côte de Liesse, Dorval, Québec, H9P 1 A5
}

$\mathbf{T}$ he Poisson's ratio, $\nu$, defines the ratio between the transverse and axial strain in a loaded material [1]. For isotropic, linear elastic materials, $\nu$ cannot be less than -1.0 nor greater than 0.5 due to empirical, work-energy, and stability considerations leading to the conditions that the shear modulus and bulk modulus have positive values [2]. Although materials with negative Poisson's ratio can exist in principle, most materials are characterized by $\nu>0$ and contract in the directions orthogonal to the applied load when they are uniaxially stretched. The discovery of materials with negative Poisson's ratio (auxetic materials, that counter intuitively expand in the transverse directions under tensile axial load) is relatively recent $[3,4]$. Auxetic response has been demonstrated in a number of natural systems, including metals with a cubic lattice [5], zeolites [6], natural layered ceramics [7] and ferroelectric polycrystalline ceramics [8]. Furthermore, following the pioneering work of Lakes [9], several periodic 2-D geometries and structural mechanisms to achieve a negative Poisson's ratio have been demonstrated. In all of these cases, careful design of the microstructure has lead to effective Poisson's ratios $\bar{\nu}<0$, despite the fact that the bulk materials are characterized by $\nu>0$. In particular, it has been shown that auxetic behavior can be achieved in a variety of highly porous materials [10], including foams with re-entrant [11-15] and chiral [16,17] microstructure, microporous polymeric materials [18], networks of rigid units [19] and skeletal structures [20]. Moreover, negative Poisson's ratio has also been shown in non-porous systems, such as laminates [21,22], sheets assemblies of carbon nanotubes [23], composites [24] and polycrystalline thin films [25].

An open area of research is within the important intermediate range between the extremes of non-porous and highly porous microstructures. Finding auxetic materials in this intermediate range greatly expands the number of structural applications, especially in those where specific porosities must be targeted. For example, in a gas turbine, there exist many perforated surfaces in the combustion chamber, the turbine section, the bypass duct, and the exhaust nozzle, which could all benefit from auxetic behavior. However, the target value for the through-thickness porosity is set by the required cooling performance or the acoustic damping function of the surface and typically ranges from $2 \%$ to $10 \%$. The design of 2-D systems capable of retaining a negative Poisson's ratio at such low values of porosity still remains a challenge [26]. Although it has been recently shown through an analytical/numerical study that diamond or star shaped perforations introduced in thin sheets can lead to auxetic behavior [27], convincing experimental evidence of a low porosity auxetic material has not been reported. The goal of this work is to demonstrate one such low porosity structure in metal via numerical simulation and material testing.

Topology optimization is a mathematical approach that enables the best design of structures that meet desired requirements [28]. Using this technique, structures that exhibit negative Poisson's ratio have been designed $[29,30]$ and the results indicate that to achieve optimal auxetic response in low porosity structures, the microstructure must comprise an array of mutually orthogonal, very elongated holes [31]. Interestingly, auxetic response has also been observed in elastomeric porous structures where a pattern of mutually orthogonal ellipses is induced by buckling [32].
Inspired by these observations, we focus on a very simple system - a square array of mutually orthogonal elliptical voids in a $2 \mathrm{D}$ metallic sheet characterized by low porosity. In particular, we investigate the effect of the hole aspect ratio on the macroscopic Poisson's ratio both through experiments and simulations. Our results demonstrate that, in this minimal system, the Poisson's ratio can be effectively controlled by changing the aspect ratio of the voids. For low aspect ratios, the structure is characterized by positive values of Poisson's ratio. However, as the aspect ratio increases, $\bar{\nu}$ is found to decrease monotonically eventually becoming negative. Remarkably, large negative values of $\bar{\nu}$ can be achieved through the adjustment of just one parameter, indicating an effective strategy for designing auxetic structures with desired porosity.

We start by exploring numerically through finite element simulations the effect of the pore aspect ratio on the macroscopic Poisson's ratio in a thin elastic plate characterized by a Young's modulus of $70 G P a$ and a Poisson's ratio of 0.35 [33]. The commercial Finite Element package ABAQUS/Standard (Simulia, Providence, RI) is used for all the simulations. To reduce the computational cost and ensure the response is not affected by boundary effects, we consider twodimensional, infinite periodic structures using representative volume elements (RVEs - see inset in Fig. 1) and periodic boundary conditions. Each mesh is constructed using six-node, quadratic, plane stress elements (ABAQUS element type CPS6). In Fig. 1-a we report the evolution of $\bar{\nu}$ as a function of the pore aspect ratio $a / b$ for linear elastic sheets with porosity $\psi=2 \%, 3 \%, 4 \%$, and $5 \%$. The results clearly show the aspect ratio $a / b$ of the holes strongly affects the lateral contraction/expansion of the structure. At aspect ratios near 1 (i.e., circular voids), the effective Poisson's ratio is nearly the same as the bulk material regardless of porosity. As the aspect ratio increases, $\bar{\nu}$ decreases and a transition from positive to negative values of $\bar{\nu}$ is observed. More precisely, for the case of structures with porosity $\psi=2 \%, 3 \%, 4 \%$, and $5 \%$ the transition from positive to negative values of $\bar{\nu}$ is observed at aspect ratios of approximately 29, 18,14 , and 11 , respectively. Thus, it appears that significant auxetic behavior can be produced in metals at very low porosity, provided the void aspect ratio $a / b$ is large enough. Finally, it is worth noting the connection among the hole aspect ratio, sheet porosity, and the length $L_{\text {min }}$ of the ligaments separating neighboring holes (see inset in Fig. 1-a)

$$
L_{\min }=\frac{L_{0}}{2}\left[1-\left(1+\frac{a}{b}\right) \sqrt{\frac{b}{a} \frac{\psi}{\pi}}\right]
$$


where $L_{0}$ denotes the size of the RVE (see Fig. 1-a). In fact, when $\bar{\nu}$ is plotted as a function of $L_{m i n} / L_{0}$ as shown in Fig. 1-b all data remarkably collapse on a single curve, which can be used to effectively design structure with the desired values of Poisson's ratio and porosity. Thus, the ligament length appears to be the essential parameter controlling the auxetic response of these structures.

Next, we proceed by attempting to reproduce this auxetic behavior experimentally. In particular, we focus on two extreme cases and investigate the response of structures with porosity $\psi=5 \%$ and aspect ratios of $a / b=1$ and $a / b=30$. The experiments were performed on a $300 \mathrm{~mm}$ by $50 \mathrm{~mm}$ by $0.4046 \mathrm{~mm}$ aluminum (6061 alloy) cellular plates (see Fig.2), which were manufactured using the CNC milling process described in Materials and Methods. The gage section of the samples were patterned with circular holes with radius of $a=b=3.154 \mathrm{~mm}$ (see Fig.2-top) and elliptical holes with major and minor axis $a=33.27 \mathrm{~mm}$ and $b=1.16 \mathrm{~mm}$ (see Fig.2bottom), respectively . Note that due to the size of the end mill (with a diameter of $0.397 \mathrm{~mm}$ ) required to manufacture the holes, the tips of exact ellipsoidal shapes were not produced, resulting in slightly lower aspect ratios, $a / b=28.7$.

The samples are tested under uniaxial tension in an Instron biaxial testing machine equipped with a $10 \mathrm{kN}$ load cell (pictures of the experimental set-up are shown in the Supporting Information). Similarly to previous studies where optical methods have been used to characterize the deformation in auxetic foams [34-37], here the displacements within the samples are captured in detail using Digital Image Correlation (DIC). DIC is a technique by which displacements can be measured by correlating (via software) the pixels in several digital images taken at different applied loads [38]. In order for multiple frames to be correlated, every single part of the image must be uniquely detectable. This requires the surface of the samples to be covered in a non-repetitive, isotropic, and high-contrast pattern. In addition, the pattern must be fine enough to capture the desired displacement details consistent with the cameras and lenses used to capture the images. In our experiments, the surfaces of the samples are painted white with a fine distribution of black speckles using a Badger 150 airbrush and water-based paint leading to a density of approximately 3-6 pixels per speckle. The deformation of the sample is monitored using a high-resolution digital camera (1.3 MPixel Retiga 1300i with a Nikon optical lens system) and given our experimental setup the displacement accuracy is estimated to be approximately 500 to $700 \mathrm{~nm}$ [38]. The samples are loaded via displacement control at a rate $0.05 \mathrm{~mm} / \mathrm{s}$ with the camera synchronized using the software Vic-Snap (Correlated Solutions) to capture images at a rate of 1 frame per second. Quantitative estimates of the deformation of the gage section of the sample are made using the image correlation software, Vic-2D (Correlated Solutions).

In addition to material testing, numerical investigations were performed on the experimental sample geometries using the nonlinear finite element code ABAQUS. Each mesh was constructed using ten-node, quadratic tetrahedral elements (ABAQUS element type C3D10) applied to the CAD model used to fabricate the samples. In order to accurately predict the material response near the voids, automatic adaptive mesh refinement is used, resulting in approximately 162,000 elements for the sample with circular voids and 119,000 elements for the sample with elliptical voids. The material is modeled as linearly elastic and perfectly plastic with a Young's modulus of $70 G P a$ and a Poisson's ratio of 0.35 [33]. The yield stress is taken to be $275 \mathrm{MPa}$ based on the experiments and is in agreement with available material data [39]. The applied experimental loading is approximated by fixing the translation at one edge and specifying a static displacement at the opposite edge. The remaining boundaries are traction free.

In Fig. 3 we present both experimental (left) and numerical (right) results for the case of circular (top) and elliptical (bottom) pores. The specimen with circular pores is shown at an applied strain of $0.34 \%$, while the specimen with elliptical holes is shown at an applied strain of $0.07 \%$. Note that the applied strain is chosen to ensure the horizontal displacements are large enough to be accurately detected by DIC. As described later, the different deformation mechanisms taking place in the two structures considered in this study result in two different values of applied strain (additional results highlighting the effect of the applied strain on $\bar{\nu}$ are shown in the Supporting Information). To minimize the effect of boundaries, we focus on the central portion of the specimen $(50 \mathrm{~mm} \times 50 \mathrm{~mm}$, see dashed red box in Fig. 2) and report contour maps for the horizontal $\left(u_{x}\right)$ and vertical $\left(u_{y}\right)$ component of the displacement field. First, an excellent agreement is observed between simulation and experimental results. Moreover, the displacement maps reported in Fig. 3 clearly show that the hole aspect ratio $a / b$ strongly affect the mechanism by which the structure deforms. For the case of circular holes, the pores are found only to locally perturb the displacement field, so that the displacement field typical of the bulk material can be easily recognized (i.e. linear distribution of $u_{x}$ and $u_{y}$ in horizontal and vertical direction, respectively). In contrast, the array of elliptical holes is found to significantly affect the displacement field, completely distorting the linear distribution of $u_{x}$ and $u_{y}$ typical of the bulk material. Finally, it is worth noticing that the nature of the displacement contours is not affected by the level of applied strain, as shown in the Supporting Information.

Focusing on $u_{x}$ we can clearly see that the material is contracting laterally for the case of circular pores. In contrast, significant lateral expansion is observed for the samples with elliptical holes demonstrating auxetic behavior. To quantify the lateral deformation we compute the effective Poisson's ratio for these structures. In both the numerical and experimental results, we sample the displacement at 8 points along each of the four boundaries of the central regions shown as a dashed box in Fig. 2. Each set of 8 points are averaged (arithmetic mean) to compute the average displacements at the boundaries: $\left\langle u_{x}\right\rangle^{L},\left\langle u_{x}\right\rangle^{R},\left\langle u_{y}\right\rangle^{T},\left\langle u_{y}\right\rangle^{B}$, where superscript $L, R$, $T$, and $B$ denote the left, right, top, and bottom boundaries, respectively. These average displacements are used to compute local strain averages

$$
\left\langle\epsilon_{x x}\right\rangle=\frac{\left\langle u_{x}\right\rangle^{R}-\left\langle u_{x}\right\rangle^{L}}{L_{0}},\left\langle\epsilon_{y y}\right\rangle=\frac{\left\langle u_{y}\right\rangle^{T}-\left\langle u_{y}\right\rangle^{B}}{L_{0}},
$$

$L_{0}=50 \mathrm{~mm}$ denoting the distance between the top/bottom and left/right boundaries in the undeformed configuration. The local strain averages are then used to calculate an effective Poisson's ratio $\bar{\nu}$ as

$$
\bar{\nu}=-\frac{\left\langle\epsilon_{x x}\right\rangle}{\left\langle\epsilon_{y y}\right\rangle}
$$

For the deformations shown in Fig. 3, the effective Poisson's ratios are reported in Table 1 where the values obtained from experiments and simulations are compared (additional numerical results for finite size samples characterized by different values of $a / b$ are reported in the Supporting Information). For the numerical cases, results for infinite periodic domains (including the same elastic-plastic behavior used for the finite sample simulations) are also reported to ensure the response is not greatly affected by the boundary conditions. First, we note an excellent agreement between experiment and simulations. Second, the results very clearly indicate that high aspect ratio ellipses lead to a material characterized by a large negative value of $\bar{\nu}$. Interestingly, the numerical results for finite and infinite size domains are very close to each other, indicating that, although the size of the samples is quite small, the effect of the boundaries is not very pronounced. (Additional numerical results for samples of different size are shown in the Supporting Information.) Therefore, our results confirm that the hole aspect ratio can be effectively used to design structures with low porosity and large negative values on $\bar{\nu}$. 
Table 1: Table summarizing the effective Poisson's ratio $\bar{\nu}$ of the two periodic structures measured from experiments and simulations.

\begin{tabular}{c|c|c|c} 
& Experiments & FEM (finite size) & FEM (infinite size) \\
\hline $\mathrm{a} / \mathrm{b}=1$ & 0.33 & 0.34 & 0.34 \\
$\mathrm{a} / \mathrm{b} \simeq 30$ & -0.73 & -0.76 & -0.65
\end{tabular}

In Fig. 4 we report numerical results to further highlight the effect of the pore aspect ratio $a / b$ on the deformation of the material. In Fig. 4- $a$ we show the deformed configuration of central region of the samples superimposed over the unloaded configuration, with the displacement field in the deformed image scaled by a factor of 100. For the circular void sample, the deformation mechanism is stretching much the same as a similar void-less structure would be. By contrast, as perviously shown in the analytical study by Grima and Gatt [27], the deformation mechanism in the elliptical void sample is mostly due to rotation, leading to a negative Poisson's ratio. Finally, the completely different mechanism by which the two structures carry the load results in very different stress distributions within the material, as shown in Fig. 4- $b$, where we report the contour map for the von Mises stress. In the structures with circular pores there are crosses that are highly stretched. These regions are yielded and deform plastically, as highlighted by the grey color in the contour map. In the case of elliptical holes, most of the structure experiences low values of stress and the deformation is found to induce rotation of the domain between holes. Stress is concentrated around the tips of the ellipses, but these can be easily reduced by carefully designing the tips to minimize the curvature.

In summary, our findings demonstrate a fundamentally new way of generating low porosity 2-D materials with negative Poisson's ratio. We show that the effective Poisson's ratio can be effectively tuned by adjusting the aspect ratio of an alternating pattern of elliptical voids. We have used numerical modeling to gain insight into the design of these structures as well as the underlying rotational mechanism causing the auxetic behavior at high void aspect ratios. These models have been verified using material testing with DIC, which has conclusively shown auxetic behavior in thin aluminum plates. We note that the structures investigated here exhibit two-dimensional

1. Greaves GN, Greer A, Lakes R, Rouxel T (2011) Poisson's ratio and modern materials. Nature materials 10:823-838.

2. Truesdell C, Noll W (2003) The Non-Linear Field Theories of Mechanics, Third Edition ed Antman S (Springer-Verlag).

3. Lakes R (1993) Advances in negative Poisson's ratio materials. Advanced Materials 5:293-296.

4. Evans KE, Alderson A (2000) Auxetic Materials: Functional Materials and Structures from Lateral Thinking. Advanced Materials 12:617-628.

5. Baughman RH, Shacklette JM, Zakhidov AA, Stafstri\%om S (1998) Negative Poisson's ratios as a common feature of cubic metals. Nature 392:362-365.

6. Grima J, Jackson R, Anderson A, Evans K (2000) Do zeolites have negative poisson's ratios? Advanced Materials 12:1912-1918.

7. Song F, Zhou J, Xu X, Xu Y, Bai Y (2008) Effect of a negative poisson ratio in the tension of ceramics. Physics Review Letters 100:245502.

8. Tan X, et al. (2009) Auxetic behavior under electrical loads in an induced ferroelectric phase. Applied Physics Letters 94:042909.

9. Lakes R (1987) Foam Structures with a Negative Poisson's Ratio. Science (New York, N.Y.) 235:1038-1040.

10. Liu Y, Hu H (2010) A review on auxetic structures and polymeric materials. Scientific Research and Essays 5:1052-1063.

11. Xu B, et al. (1999) Making negative poisson's ratio microstructures by soft lithography. Adv. Mat. 11:1186-1189.

12. Smith C, Grima J, Evans $K$ (2000) A novel mechanism for generating auxetic behaviour in reticulated foams: missing rib foam model. Acta Materialia 48:4349-4356.

13. Larsen U, Sigmund O, Bouwstra S (1997) Design and fabrication of compliant mechanisms and material structures with negative poissoni\%॰s ratio. Journal of Microelectromechanical Systems 6:99-106.

14. Schwerdtfeger J, et al. (2012) Mechanical characterisation of a periodic auxetic structure produced by sebm. Physica Status Solidi B 7:1347i\% 1352. cubic symmetry, meaning that the effective response is anisotropic. While further investigation of material symmetry is outside the scope of the current work, it is an important design issue. Thus, this work provides not only a guide for the simple design of auxetic materials but serves as a basis for future investigations into such areas as material symmetry and void shape optimization to minimize stress concentrations while maintaining a desired Poisson's ratio.

\section{Materials and Methods}

Materials. Test samples were cut from 6061 Aluminum alloy $0.4046 \mathrm{~mm}$ thick plates. The bulk material has a Young's modulus of $70 \mathrm{GPa}$, Poisson's ratio of 0.35 , and yield stress of $275 M P a$. In the numerical simulations its behavior is modeled as linearly elastic and perfectly plastic.

Fabrication. Test samples were fabricated using a Haas OM-2A CNC Machine together with a 2-flute ultra-duty coated (TiCn) carbide end mill (diameter $=0.39687 \mathrm{~mm}$ ). Samples were fed intothe mill at a speed of 2 inches per minute with a 0.003 inch depth of cut. Samples were designed in Solidworks CAD software (Dassault Systems) and imported to the CNC machine via SolidCAM.

Testing. Samples were tested at the MIT Impact and Crashworthiness Lab. Prior to testing, samples were coated in white water-based paint using a Badger150 airbrush. Contrast was added by spraying a fine pattern of black waterbased paint to the recorded surface using the same airbrush. For the tests, samples were loaded into an Instron bi-axial testing machine equipped with a 10 $k N$ load cell using $5 \mathrm{~cm}$ wedge grips. The grips were secured using a steel block with aluminum spacers screwed together at a fixed torque resulting in constant contact pressure. Samples were illuminated uniformly in situ by means of tripod mounted diffused isotropic lighting. A Retiga 1300i camera (Nikon optical lens system) was mounted to a tripod and focused on a $80 \mathrm{~mm}$ by $60 \mathrm{~mm}$ rectangle in the central gage section of the samples providing digital imagery throughout the tests. The original image was then cropped to the $50 \mathrm{~mm}$ by $50 \mathrm{~mm}$ dimensions used in this study. The camera has a 1.3 MPixel resolution resulting in a pixel size of $6.7 \mu \mathrm{m}$ by $6.7 \mu \mathrm{m}$. Samples were tested in tension at a rate of $0.05 \mathrm{~mm} / \mathrm{s}$ with cameras triggered externally (via Vic-Snap) to capture 1 frame per second in synchronization with the applied Instron loading.

ACKNOWLEDGMENTS. This work has been funded by Rolls-Royce Energy. M.T., L.F. and K.B. thank Prof. Tomasz Wierzbicki and Stephane Marcadet (MIT Impact and Crashworthiness Lab) for their support with the experiments.

15. Scarpa F, Panayiotou P, Tomlinson G (2000) Numerical and experimental uniaxial loading on in-plane auxetic honeycombs. The Journal of Strain Analysis for Engineering Design 35:383-388.

16. Prall D, Lakes R (1996) Properties of a chiral honeycomb with a poisson's ratio of -1. International Journal of Mechanical Sciences 39:305-314.

17. Spadoni A, Ruzzene M (2012) Elasto-static micropolar behavior of a chiral auxetic lattice. Journal of the Mechanics and Physics of Solids 60:156-171.

18. Caddock B, Evans K (1989) Microporous materials with negative poisson's ratios. i. microstructure and mechanical properties. Journal of Physics D: Applied Physics 22:1877-1882.

19. Grima J, Alderson A, Evans $K$ (2005) Auxetic behaviour from rotating rigid units. Physica Status Solidi (b) 242:561-575.

20. Mitschke H, et al. (2011) Finding auxetic frameworks in periodic tessellations. Adv. Mat. 23:2669-2674.

21. Milton G (1992) Composite materials with poisson's ratios close to -1 . Journal of the Mechanics and Physics of Solids 40:1105-1137.

22. Herakovich C (1984) Composite laminates with negative through-the-thickness poisson's ratios. Journal of Composite Materials 18:447-455.

23. Hall L, et al. (2008) Sign change of poisson's ratio for carbon nanotube sheets. Science 320:504-507.

24. Kochmann D, Venturini G (2013) Homogenized mechanical properties of auxetic composite materials in finite-strain elasticity. Smart Materials and Structures 22:084004.

25. Mullen R, Ballarini R, Yin Y, Heuer A (1997) Monte carlo simulation of effective elastic constants of polycrystalline thin films. Acta Materialia 45:2247-2255.

26. Prawoto $Y$ (2012) Seeing auxetic materials from the mechanics point of view: A structural review on the negative poisson's ratio. Computational Materials Science 58:140-153.

27. Grima J, Gatt $\mathbf{R}$ (2010) Perforated sheets exhibiting negative poisson's ratios. Advanced Engineering Materials 12:460-464. 
28. Bendsoe M, Sigmund O (2003) Topology Optimization: Theory, Methods and Applications (Springer).

29. Larsen U, Sigmund O, Bouwstra S (1997) Design and fabrication of compliant micromechanisms and structures with negative poisson's ratio. Journal of Microelectromechanical systems 6:99-106.

30. Schwerdtfeger J, et al. (2011) Design of auxetic structures via mathematical optimization. Adv. Mat. 23:2650-2654.

31. Sigmund O (1994) Design of material structures using topology optimization (Ph.D. thesis, Technical University of Denmark).

32. Bertoldi K, Reis PM, Willshaw S, Mullin T (2010) Negative poisson's ratio behavio induced by an elastic instability. Adv. Mat. 22:361-366.

33. Tilley J (2004) Understanding Solids: The Science of Materials (Wiley).

34. Chen C, Lakes R (1991) Holographic study of conventional and negative poisson's ratio metallic foams: elasticity, yield and micro-deformation. Journal of Materials Science 26:5397-5402.
35. McDonald S, Ravirala N, Withers P, Alderson A (2009) In situ three-dimensional x-ray microtomography of an auxetic foam under tension. Scripta Materialia 60:232-235.

36. Pierron F (2010) Identification of poisson's ratios of standard and auxetic low density polymeric foams from full-field measurements. Journal of Strain Analysis for Engineering Design 45:233-253.

37. Pierron F, et al. (2013) Comparison of the mechanical behaviour of standard and auxetic foams by $x$-ray computed tomography and digital volume correlation. Strain. doi: 10.1111/str.12053.

38. Sutton MA, Orteu JJ, Schreier H (2009) Image Correlation for Shape, Motion and Deformation Measurements (Springer).

39. Ashby M, Jones D (1996) Engineering Materials 1: An Introduction to their Properties and Applications, 2nd Ed. (Butterworth-Heinemann). 

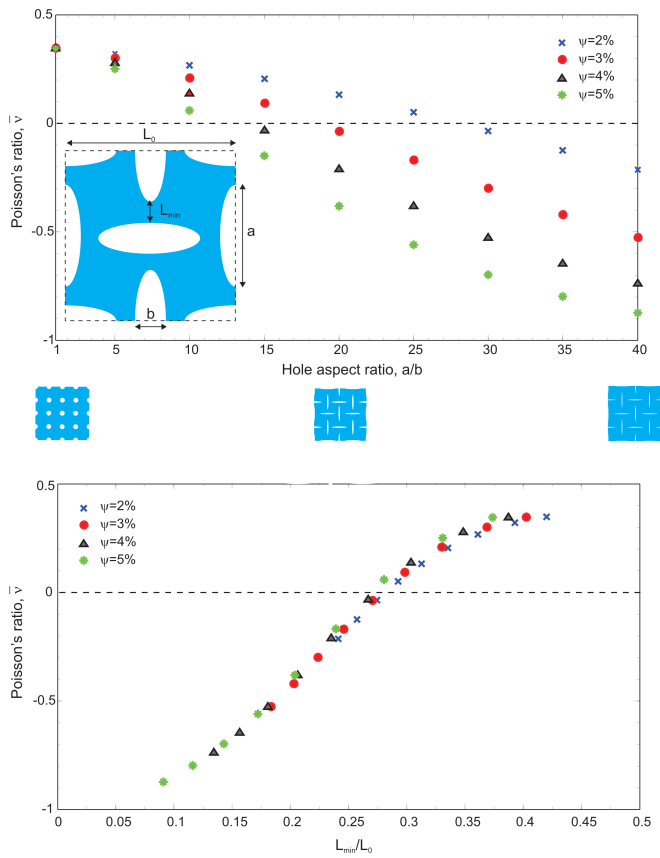

Fig. 1: (a): Results of the numerical investigation on the effect of the hole aspect ratio $a / b$ for an infinite periodic square array in an elastic matrix. Four different values of porosity are considered. The RVE considered in the analysis is shown as an inset. (b): All data collapse on a single curve when $\bar{\nu}$ is plotted as a function of $L_{m i n} / L_{0}$.
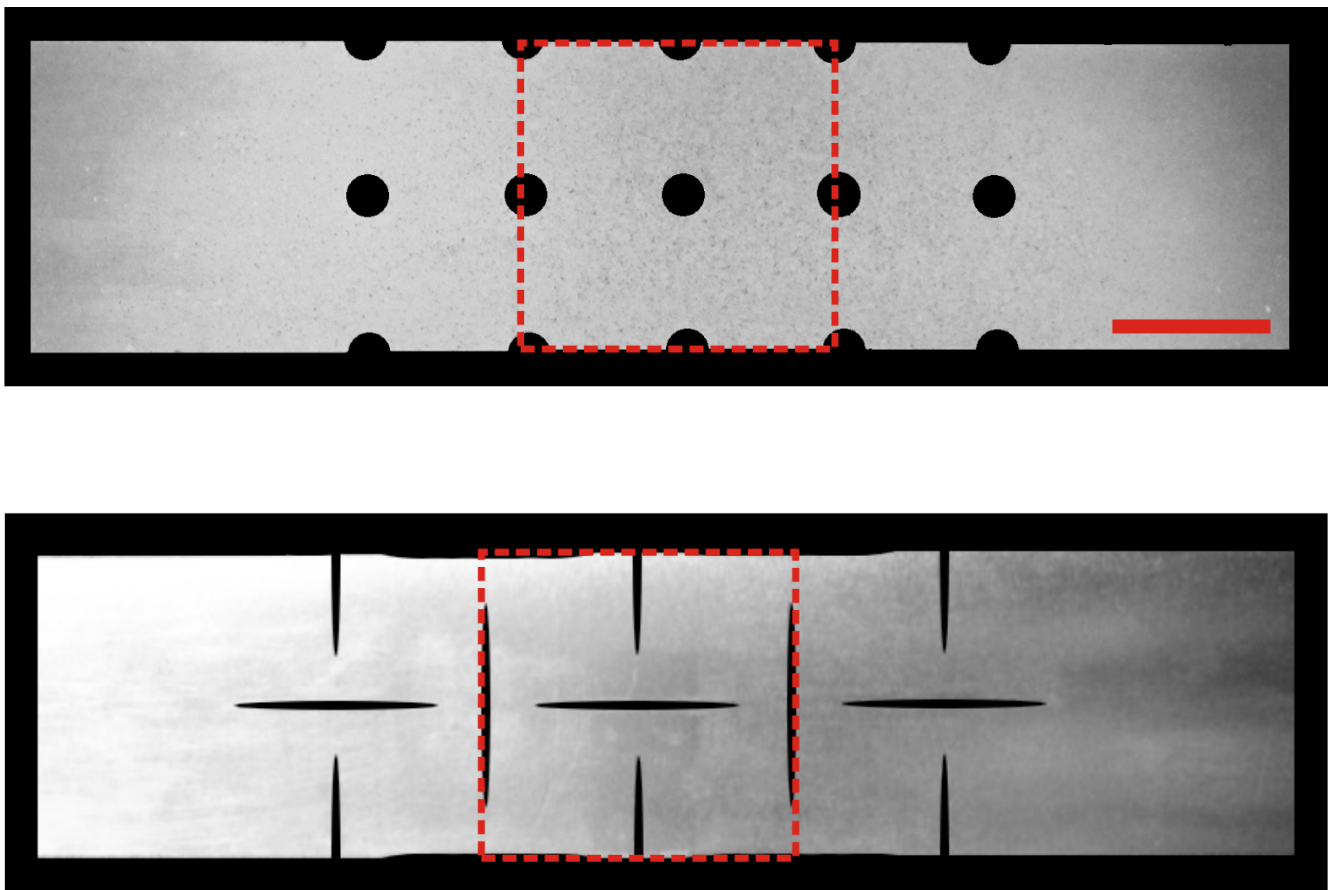

Fig. 2: Samples comprising of a square array of (top) circular and (bottom) elliptical (with $a / b \cong 30$ ) holes in the undeformed configuration. The dashed rectangle represents region over which we perform the ensemble averaging. (Scale bar: $25 \mathrm{~mm}$ ) 


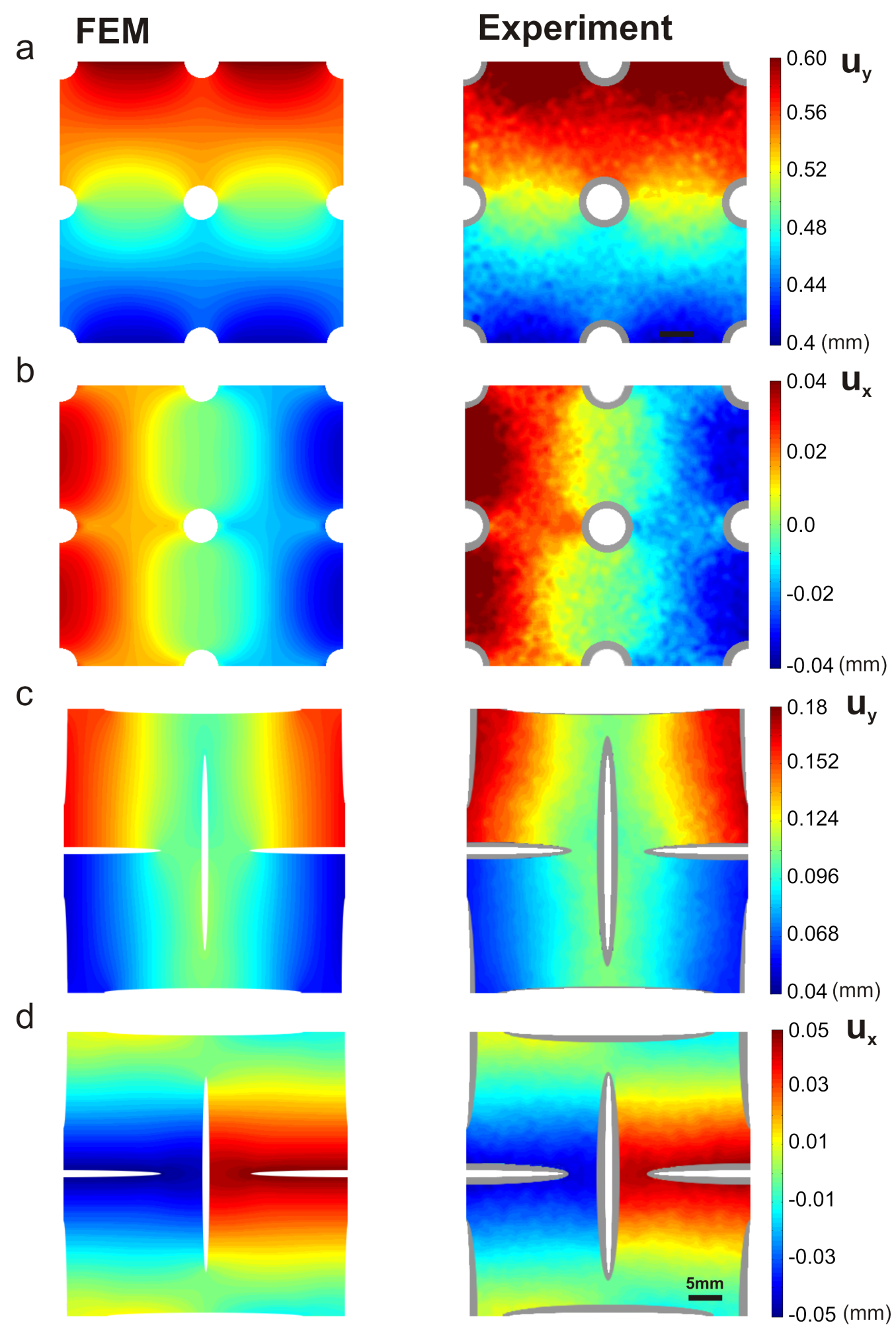

Fig. 3: Contour maps for the horizontal $\left(u_{x}\right)$ and vertical $\left(u_{y}\right)$ component of the displacement field. Numerical (left) and experimental (right) results are quantitatively compared, showing excellent agreement. In (a) and (b), the applied strain is $0.34 \%$, while in (c) and (d), the applied strain is $0.07 \%$. Note that gray areas on experimental results show regions where DIC data could not be obtained. 


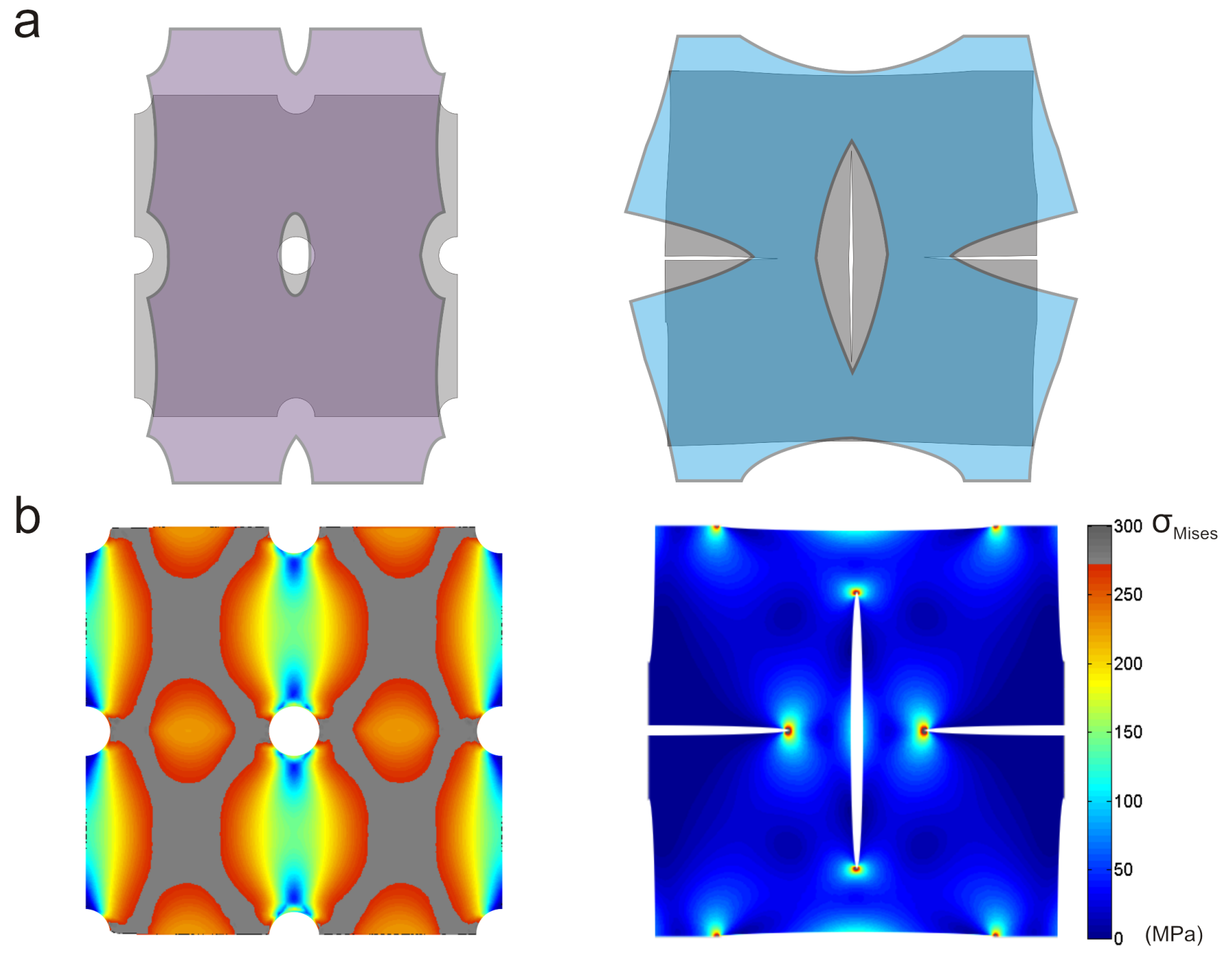

Fig. 4: Effect of the pore aspect ratio $a / b$ on the deformation of the structure. (a) Deformed configuration superimposed over the unloaded configuration, with the displacement field in the deformed image scaled by a factor of 100 . (b) Von Mises stress distribution with plastified areas colored gray. The applied strain is $0.34 \%$ and $0.07 \%$ for the structure with circular and elliptical holes, respectively. 